\title{
Revealing the parameters of the open cluster Ruprecht $58^{\star}$
}

\author{
E. Giorgi ${ }^{\mathrm{a}, 1}$, R. A. Vázquez ${ }^{\mathrm{a}, 1}$, G. R. Solivella ${ }^{\mathrm{a}, 1}$, \\ R. B. Orellana ${ }^{a}$, \\ ${ }^{a}$ Facultad de Ciencias Astronómicas y Geofísicas UNLP, IALP-CONICET, Paseo \\ del Bosque s/n, 1900, La Plata, Argentina \\ and \\ J. Nuñez ${ }^{b}$ \\ ${ }^{\mathrm{b}}$ Facultad de Ingeniería, CIBA, Universidad Nacional de Jujuy, Gorriti 237, 4600, \\ S. S. de Jujuy, Argentina
}

\begin{abstract}
We present results of a study that combines $U B V I$ photometry, MK spectral classification and proper motions in the area of the, up to now unknown, open cluster Ruprecht 58 at the Puppis region. Star counts from the 2MASS data catalog together with the analysis of CCD $U B V I$ photometry demonstrate that it is a real open cluster with $9^{\prime}$ size approximately. The cluster is placed at a distance of 3.9 $\mathrm{kpc}$ and is about $250 \mathrm{Myr}$ old with mean reddening $E_{(B-V)}=0.33$ mag. Proper motions confirm Ruprecht 58 is a real cluster with mean absolute proper motions $\mu_{\alpha} \cos \delta=-2.77 \pm 0.45 \mathrm{mas} / \mathrm{yr}$ and $\mu_{\delta}=4.54 \pm 0.45 \mathrm{mas} / \mathrm{yr}$ in the magnitude range $13.5<V<14.5$ and $\mu_{\alpha} \cos \delta=-2.70 \pm 0.32 \mathrm{mas} / \mathrm{yr}$ and $\mu_{\delta}=3.19 \pm 0.32 \mathrm{mas} / \mathrm{yr}$ in the range $14.5<V<16.0$. The computation of the cluster mass spectrum slope yielded $x=1.8$ in the mass range from $\approx 1.4$ to $\approx 4 m_{\odot}$.
\end{abstract}

Key words: Open clusters and associations; individual: Ruprecht 58 methods: statistical - stars: kinematics - stars: luminosity function, mass function - stars: Hertzsprung-Russell (H-R) and C-M diagrams

\footnotetext{
^ Based on observations collected at the Complejo Astronómico El Leoncito (CASLEO), Argentina.

1 Visiting astronomer, Complejo Astronómico El Leoncito operated under agreement between the Consejo Nacional de Investigaciones Científicas y Técnicas de la República Argentina and the National Universities of La Plata, Córdoba and San Juan
} 


\section{Introduction}

The detection of the Canis Major (CMa) over-density by Martin et al. (2004) produced a renaissance of interest for the Third Galactic Quadrant $\left(180^{\circ}<\right.$ $l<270^{\circ}$ ) of the Milky Way. The vivid debate in the last year (Momany et al. 2004, Bellazzini et al. 2004) on this over-density clearly demands a better picture of the Galaxy structure in this region. Young open clusters are recognized as ideal spiral arm tracers (Becker \& Fenkart 1970, Feinstein 1994) as they are near the spiral arm in which they formed. Surprisingly, the shape and extent of the Perseus and Cygnus-Norma arms in the Third Quadrant are far from being clear and settled. Russeil (2003) using star forming complexes finds that both the Perseus and Norma-Cygnus arms are not visible at all in the Third Quadrant, confirming previous results by May et al. (1997). Nevertheless, they could confirm previous suggestions about the shape and location of the Galactic warp and show how bridges of material are present in a few anti-center directions. To date, no study has probed the spiral structure of the Third Galactic Quadrant using young star clusters which are vital to better trace the spiral pattern in largely overlooked region of the Galaxy. Additionally, open clusters are not only the main source of stellar enrichment in our galaxy but also the kind of objects through which the theories of star formation and evolution can be adequately addressed; they offer a chance to know the probable spatial variations in the star formation rates (Scalo 1986) and give information regarding the history and evolution of the galactic disk (e.g. Janes \& Adler 1982; Massey et al. 1995).

For the last several years our group has been conducting a systematic, homogeneous and accurate $U B V R I$ photometric survey of open clusters in this part of the Galaxy [see Moitinho (2001), Giorgi et al. (2005, and references therein), Baume et al. (2004), Carraro et al. (2005a, and references therein)] with special emphasis in Vela-Puppis. Our target on this occasion is Ruprecht 58 , an open cluster that WEBDA sets at $\alpha_{2000}=8^{h} 14^{m} 7^{s}$ and $\delta_{2000}=-31^{\circ} 57^{\prime}$ $\left(l=250.4^{\circ}, b=+1.6^{\circ}\right)$ but for which we found no information in any existing data bases. Lindoff (1968) reports $B V$ photographic photometry down to $V=15.2 \mathrm{mag}$ which could not be compared to ours because of no stellar identification in his work. Anyway, Lindoff's conclusion is that no open cluster exists down to his limiting magnitude.

In Sect. 2 we describe the observation routines and the reduction processes. The analysis of the photometric and of spectroscopic data, the reddening discussion, membership, distance and age can be found in Sect. 3. The proper motion analysis is presented in Sect. 4. The cluster mass spectrum is determined in Sect. 5 and the conclusions are given in Sect. 6. 


\section{Observations}

\subsection{Photometry}

CCD observations in the $U B V I$ system were carried out in the field of Ruprecht 58 on the nights of December 24 and 25, 2003, at the Complejo Astronómico El Leoncito, CASLEO, Argentina, using the 2.15-m telescope equipped with a CCD ROPER 1300B, $1340 \times 1300$ and 0.226 " / pix scale, covering $4.2^{\prime}$ on a side. Exposure times were of 300 seconds in $U ; 300,50$ and 5 seconds in $B$; 300, 30 and 3 seconds in $V$, and 120, 60 and 3 seconds in $I$ under seeing values ranging from $1.3^{\prime \prime}$ to $2.1^{\prime \prime}$.

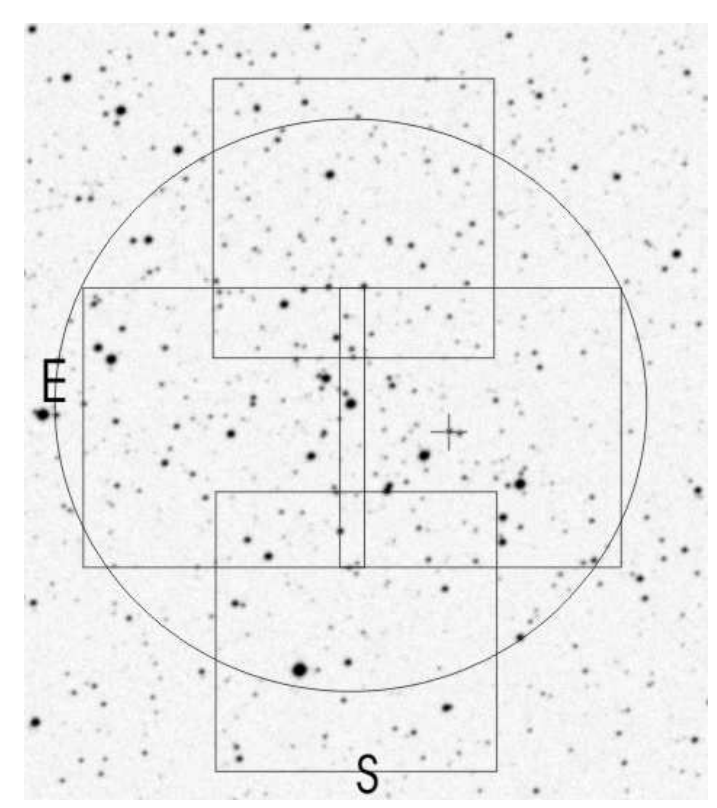

Fig. 1. Finding chart of Ruprecht 58 adapted from a DSS image. The circle is the computed angular size of the cluster $9^{\prime}$ approximately (centered at $\left.\alpha_{2000}=8^{h} 14^{m} 50^{s}, \delta_{2000}=-31^{\circ} 56^{\prime} 29^{\prime \prime}\right)$; the squares show the four science frames observed at CASLEO. North is at top and East at left. The plus sign is the cluster center as given in WEBDA

Instrumental magnitudes were obtained by means of the point spread function (PSF) fitting using the DAOPHOT (Stetson 1987) package within IRAF $^{2}$ and tied to the standard UBVI system with several standard stars of Landolt (1992) list and secondary standards in the cluster NGC 2571 previously calibrated with Landolt standards as well. A description of the reduction procedure can be found in Giorgi et al. (2002). Mean extinction coefficients at CASLEO, 0.49 in $U, 0.27$ in $B, 0.12$ in $V$ and 0.03 in $I$, were applied; the

$\overline{2}$ IRAF is distributed by NOAO, which are operated by AURA under cooperative agreement with the NSF 
transformation equations to the standard system were of the form:

$$
\begin{aligned}
& u=U+u_{1}+u_{2} \times X+u_{3} \times(U-B) \\
& b=B+b_{1}+b_{2} \times X+b_{3} \times(B-V) \\
& v=V+v_{1}+v_{2} \times X+v_{3} \times(B-V) \\
& i=I+i_{1}+i_{2} \times X+i_{3} \times(V-I)
\end{aligned}
$$

where $u_{2}, b_{2}, v_{2}$ and $i_{2}$ are the extinction coefficients for the $U B V I$ bands respectively, $X$ the air masses of each exposure and $u_{1}, b_{1}, v_{1}, i_{1}, u_{3}, b_{3}, v_{3}$, and $i_{3}$ the fitting constants values.

The area covered in the current survey is shown in Fig. 1 where each square superposed onto the Digitized Sky Survey plate, DSS, represents the CCD frame. Table 1 lists the typical DAOPHOT errors found at different $V$ magnitude ranges.

Table 1

Daophot errors as function of the $V$ magnitude

\begin{tabular}{ccccc}
\hline \hline V range & $\epsilon_{V}$ & $\epsilon_{(B-V)}$ & $\epsilon_{(U-B)}$ & $\epsilon_{(V-I)}$ \\
\hline $10-14$ & 0.02 & 0.02 & 0.03 & 0.02 \\
$14-16$ & 0.02 & 0.03 & 0.04 & 0.03 \\
$16-18$ & 0.02 & 0.03 & 0.05 & 0.09 \\
$18-20$ & 0.02 & 0.03 & 0.10 & - \\
$>20$ & 0.04 & 0.08 & - & - \\
\hline \hline
\end{tabular}

Table 2 (available at CDS) contains the $\mathrm{x}-\mathrm{y}$ star positions and the photometric output including $V$ magnitudes and $B-V$ colors for 1117 stars, $U-B$ for 654 stars, and $V-I$ for 1075 stars. For a reduction of the uncertainties level, no star with DAOPHOT errors larger or equal than 0.10 mag have been considered in any further analysis. An abbreviated version of Table 2 is given below.

\subsection{Spectroscopy}

Spectral data for 13 of the brightest cluster stars were collected at the 2.15$\mathrm{m}$ telescope of CASLEO (Argentina) during two observing runs in March 11-14 and April 1-4, 2004. The spectra were obtained with the REOSC-DS Cassegrain spectrograph and a Tek $1024 \times 1024$ detector using a $600 \mathrm{l} / \mathrm{mm}$ grating in the first order. The spectra have a wavelength range of 3900-5500 $\AA$ (the traditional spectral region for classification from CaII K line to $\mathrm{H} \beta$, allowing a precise MK type), $2.5 \AA$ /pixel dispersion $(\approx 1800$ resolution) and were reduced using standard procedures with IRAF. For the clasification purposes we used MK standard stars taken with the same configuration at CASLEO and 
Table 2

Photometry, astrometry, proper motions and membership for stars in the field of Ruprecht 58 (abbreviated version)

\begin{tabular}{|c|c|c|c|c|c|c|c|c|c|c|c|c|}
\hline ID & $\mathrm{x}$ & $\mathrm{y}$ & $\alpha_{(2000)}$ & $\delta_{(2000)}$ & $\mathrm{V}$ & B-V & U-B & V-I & $\begin{array}{c}\mu_{\alpha} \\
(\mathrm{mas} / \mathrm{y})\end{array}$ & $\begin{array}{c}\mu_{\delta} \\
(\mathrm{mas} / \mathrm{y})\end{array}$ & Rem. & $\mathrm{P}(\%)$ \\
\hline 1 & 880.92 & -386.42 & 123.72478360 & -32.01410950 & 10.10 & 0.90 & 0.93 & 1.03 & -4.10 & -2.70 & & 0.00 \\
\hline 2 & 1895.48 & 399.01 & 123.65309450 & -31.96508480 & 11.43 & 0.06 & 0.32 & 0.02 & -6.60 & 1.90 & & 0.00 \\
\hline 3 & 100.80 & 1027.29 & 123.78266270 & -31.92884730 & 11.72 & 0.23 & 0.16 & 0.17 & -4.30 & -1.40 & & 0.00 \\
\hline 4 & 1474.87 & 543.16 & 123.68346560 & -31.95677750 & 11.56 & 0.39 & 0.20 & 0.42 & 0.30 & 3.00 & & 0.00 \\
\hline 5 & 1158.19 & 786.31 & 123.70649620 & -31.94233560 & 11.90 & 1.10 & 1.07 & 1.26 & -8.10 & 4.30 & & 0.00 \\
\hline 6 & 1052.24 & 903.29 & 123.71425950 & -31.93531280 & 12.42 & 0.28 & 0.13 & 0.35 & -0.40 & -4.40 & & 0.00 \\
\hline 7 & 44.14 & 1081.63 & 123.78679360 & -31.92560030 & 12.62 & 0.16 & 0.01 & 0.13 & -0.40 & 2.50 & & 0.00 \\
\hline 8 & 1108.14 & 1807.24 & 123.71152770 & -31.88016200 & 12.73 & 1.32 & 1.32 & 1.50 & -2.50 & 10.60 & & 0.00 \\
\hline 9 & 880.11 & 1239.97 & 123.72706980 & -31.91499840 & 12.79 & 0.71 & 0.22 & 0.87 & -8.10 & 3.20 & & 0.00 \\
\hline 10 & 971.58 & 561.10 & 123.72178947 & -31.95647382 & 12.90 & 1.00 & 0.75 & 1.26 & & & & \\
\hline 11 & 764.10 & 124.81 & 123.73386920 & -31.98309870 & 12.91 & 0.70 & 0.34 & 0.77 & -31.70 & 10.00 & & 0.00 \\
\hline 12 & 618.70 & 674.39 & 123.74502950 & -31.94975560 & 13.00 & 1.05 & 0.76 & 1.27 & -2.70 & 5.30 & & 0.00 \\
\hline 13 & 1812.40 & 252.92 & 123.65884560 & -31.97406980 & 13.02 & 0.46 & 0.07 & 0.60 & -7.00 & 10.10 & & 0.00 \\
\hline 14 & 1524.46 & -582.18 & 123.68308780 & -32.02507050 & 13.07 & 0.56 & 0.11 & 0.63 & & & & \\
\hline 15 & 1805.79 & 143.99 & 123.66339442 & -31.98150036 & 13.25 & 0.52 & 0.08 & 0.67 & & & & \\
\hline 16 & 1302.21 & 391.29 & 123.69864540 & -31.96666266 & 13.28 & 1.07 & 0.86 & 1.34 & & & & \\
\hline 17 & 318.79 & 556.76 & 123.76638830 & -31.95726670 & 13.50 & 0.38 & 0.22 & 0.45 & -4.10 & 3.20 & & 0.00 \\
\hline 18 & 1106.17 & 1082.41 & 123.71063890 & -31.92434230 & 13.55 & 0.50 & 0.05 & 0.59 & -8.10 & 6.50 & & 0.00 \\
\hline 19 & 1097.09 & -362.09 & 123.70930950 & -32.01235750 & 13.64 & 1.35 & 1.79 & 1.53 & -3.70 & -0.80 & & 0.00 \\
\hline 20 & 672.28 & 200.23 & 123.74053330 & -31.97858920 & 13.66 & 0.29 & 0.17 & 0.36 & 2.30 & -1.50 & $\mathrm{~m}$ & 0.06 \\
\hline 21 & 605.00 & -79.74 & 123.74501770 & -31.99571230 & 13.70 & 1.15 & 1.23 & 1.34 & 6.00 & -0.10 & & 0.00 \\
\hline 22 & 1086.30 & 221.64 & 123.71086210 & -31.97681370 & 13.74 & 0.29 & 0.15 & 0.34 & -0.50 & 1.20 & $\mathrm{~m}$ & 0.62 \\
\hline 23 & 1314.29 & 416.52 & 123.69779980 & -31.96514898 & 13.74 & 0.94 & 0.74 & 1.15 & & & & \\
\hline 24 & 596.07 & -770.69 & 123.74807520 & -32.03638110 & 13.80 & 0.69 & 0.26 & 0.83 & & & & \\
\hline 25 & 1344.73 & 858.54 & 123.69566860 & -31.93862748 & 13.86 & 0.26 & 0.09 & 0.35 & & & $\mathrm{~m}$ & \\
\hline 26 & 1080.61 & 1308.93 & 123.71283360 & -31.91056500 & 13.86 & 0.56 & 0.09 & 0.71 & -3.60 & 2.90 & & 0.77 \\
\hline 27 & 1541.37 & -53.59 & 123.67786180 & -31.99304390 & 13.90 & 1.02 & 0.86 & 1.21 & -4.50 & 3.90 & & 0.00 \\
\hline 28 & 969.86 & 1301.44 & 123.72076210 & -31.91114390 & 13.93 & 0.30 & 0.12 & 0.43 & -0.70 & 6.00 & $\mathrm{~m}$ & 0.72 \\
\hline 29 & 1590.77 & 644.44 & 123.67529650 & -31.95047140 & 13.93 & 0.23 & 0.09 & 0.29 & 5.50 & 3.00 & $\mathrm{~m}$ & 0.05 \\
\hline 30 & 1238.27 & 1305.48 & 123.70152270 & -31.91059810 & 13.94 & 0.28 & 0.11 & 0.40 & -0.50 & 3.80 & $\mathrm{~m}$ & 0.76 \\
\hline 31 & 1009.34 & 2132.93 & 123.71914620 & -31.86216420 & 13.96 & 1.02 & 0.89 & 1.19 & & & & \\
\hline 32 & 2210.73 & 46.93 & 123.62998560 & -31.98617980 & 14.00 & 0.52 & 0.14 & 0.68 & -4.70 & 7.80 & & 0.21 \\
\hline 33 & 795.72 & 2112.69 & 123.73409940 & -31.86337860 & 14.11 & 0.46 & 0.17 & 0.58 & & & & \\
\hline 34 & 34.18 & 1275.52 & 123.78740750 & -31.91360880 & 14.17 & 0.30 & 0.18 & 0.33 & & & $\mathrm{~m}$ & \\
\hline 35 & 1454.71 & 1478.02 & 123.68797030 & -31.90145862 & 14.18 & 0.28 & 0.11 & 0.39 & & & $\mathrm{~m}$ & \\
\hline 36 & 881.73 & 1971.47 & 123.72807900 & -31.87185180 & 14.19 & 0.80 & 0.53 & 0.91 & & & & \\
\hline 37 & 377.72 & 716.87 & 123.76237270 & -31.94743730 & 14.24 & 0.28 & 0.18 & 0.33 & -5.80 & 3.70 & $\mathrm{~m}$ & 0.54 \\
\hline 38 & 108.46 & 751.40 & 123.78173800 & -31.94564590 & 14.24 & 0.68 & 0.26 & 0.78 & -17.60 & 5.50 & & 0.00 \\
\hline 39 & 154.97 & 1160.24 & 123.77894590 & -31.92068060 & 14.24 & 1.34 & 1.48 & 1.60 & -3.30 & 4.80 & & 0.62 \\
\hline 40 & 341.78 & 1067.31 & 123.76543740 & -31.92612840 & 14.26 & 0.26 & 0.16 & 0.29 & -11.50 & -5.10 & $\mathrm{~m}$ & 0.00 \\
\hline
\end{tabular}

Note: star coordinates are in degrees and proper motions in mas/y. (::) denotes photometric errors $\epsilon_{\text {phot }}>0.1$. The proper motion $\mu_{\alpha}$ is given at the cluster declination.

Rem: $m$ shows probable cluster members according to the photometric criterion explained in Sect. 3.

Last column gives the star probability according our proper motions analysis.

Some stars were labeled PME (probable member by extension) and NME (non-member by extension) from an extrapolation procedure using the parameters derived in Sect. 4. The complete table can be found in the electronic edition of New Astronomy too

the Digital Spectra Classification of R.O. Gray ${ }^{3}$ and the MK Standard ${ }^{4}$.

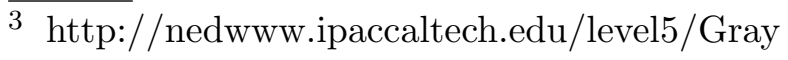




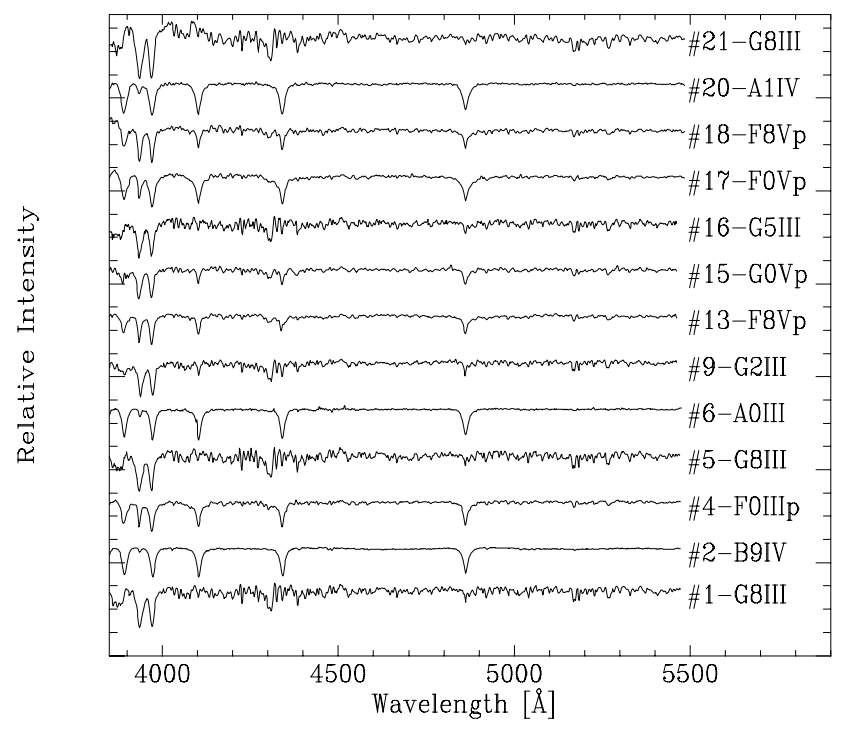

Fig. 2. Spectra of 13 stars grouped by our classification given in Table 3

Spectra and the respective spectral classification are shown in Fig. 2. It is worth mentioning that for a number of stars we found discrepancies in the intensities of the $\mathrm{K}$ CaII lines and the spectral types assigned according to the $\mathrm{H}$ lines in the sense that $\mathrm{K}$ CaII lines show intensities corresponding to spectral earlier than according to the $\mathrm{H}$ lines. These stars compose the $38 \%$ (two of them are giants; other three of the main sequence) of the total stars with spectral types and we assume them peculiar stars; the others constitute the $61 \%$ and are are of giant types. The results and description of peculiarities are given in column 9 of Table 3 . We are confident that our classification is not wrong for more than one sub-type and that the luminosity class is precise.

\section{Data Analysis}

\subsection{Cluster size}

The reliability of Ruprecht 58 and its spatial extension were analyzed using 2MASS data of all stars included inside a $30^{\prime} \times 30^{\prime}$ box centered in the cluster published coordinates. The technique is based in star counts that were performed in a series of successive rings $1^{\prime}$ width from the cluster center as done in Baume et al. (2004), Carraro et al. (2005a)]. Counts in each ring were divided by the ring area to construct the density profile. During this process we noticed some sort of count fluctuations that we assume are due to the presence of patches of dust across the cluster surface. So, several attempts had to

$\overline{4}$ http://stellar.phys. appstate.edu/Standards 


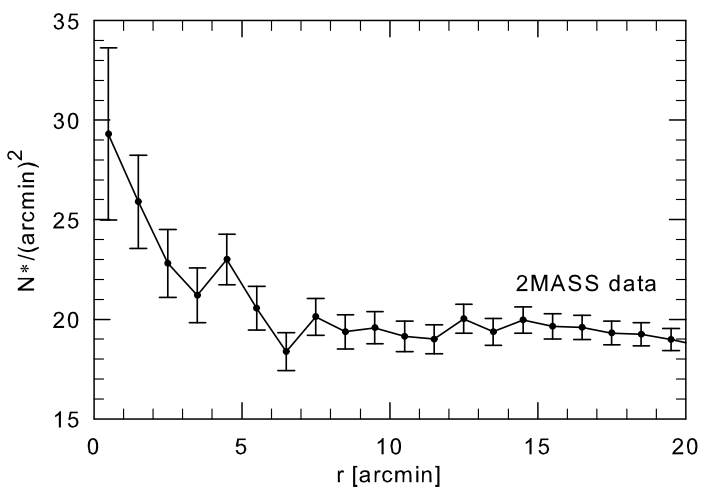

Fig. 3. The star density as a function of the distance to the center of the cluster computed with 2MASS as explained in the text. Bars are the $\sqrt{N}$ counts

be done until a clean star density peak was found at $\alpha_{2000}=8^{h} 14^{m} 50^{s}$ and $\delta_{2000}=-31^{\circ} 56^{\prime} 29^{\prime \prime}$. That sets a new cluster center slightly shifted from the original one given in WEBDA. The density profile in Fig. 3 reveals an evident stellar over-density that extends up to $r=4.5^{\prime} \pm 1^{\prime}$ where it merges with the stellar density of background. This value is adopted here as the cluster radius. Comparing to the area covered by our photometric survey shown in Fig. 1 we are confident that we have surveyed $\approx 75 \%$ of the whole cluster.

\subsection{Cluster members, reddening, distance and age of Ruprecht 58}

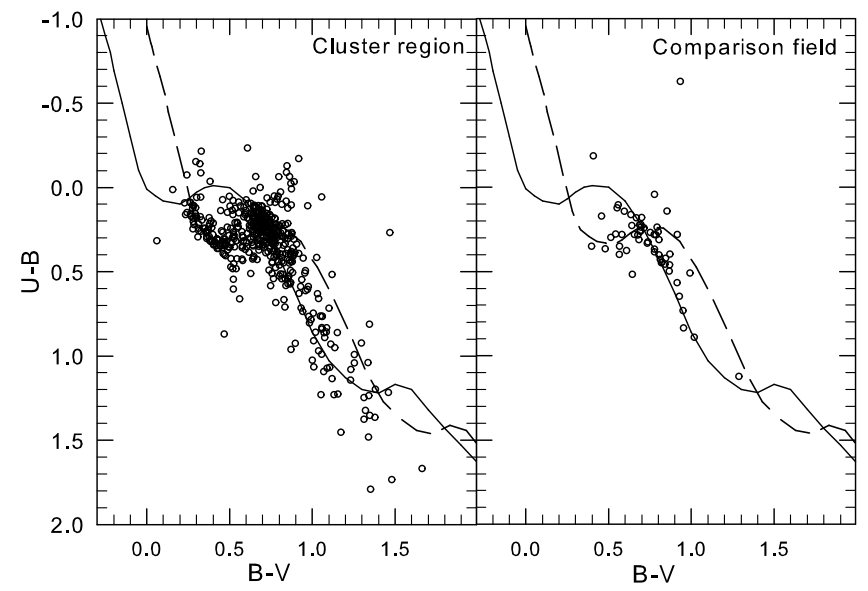

Fig. 4. Left panel: TCD of all stars inside the cluster radius. The continuous lines show the intrinsic loci for main sequence stars (Schmidt-Kaler 1982) in the normal position and the dashed one shifted by $E_{(B-V)}=0.33$ and $E_{(U-B)}=0.29$ (§3.2). Right panel: Idem for the comparison field stars

Figures 4 and 5 show the $U-B / B-V$ two-color (TCD) and the $V /(B-V)$, 


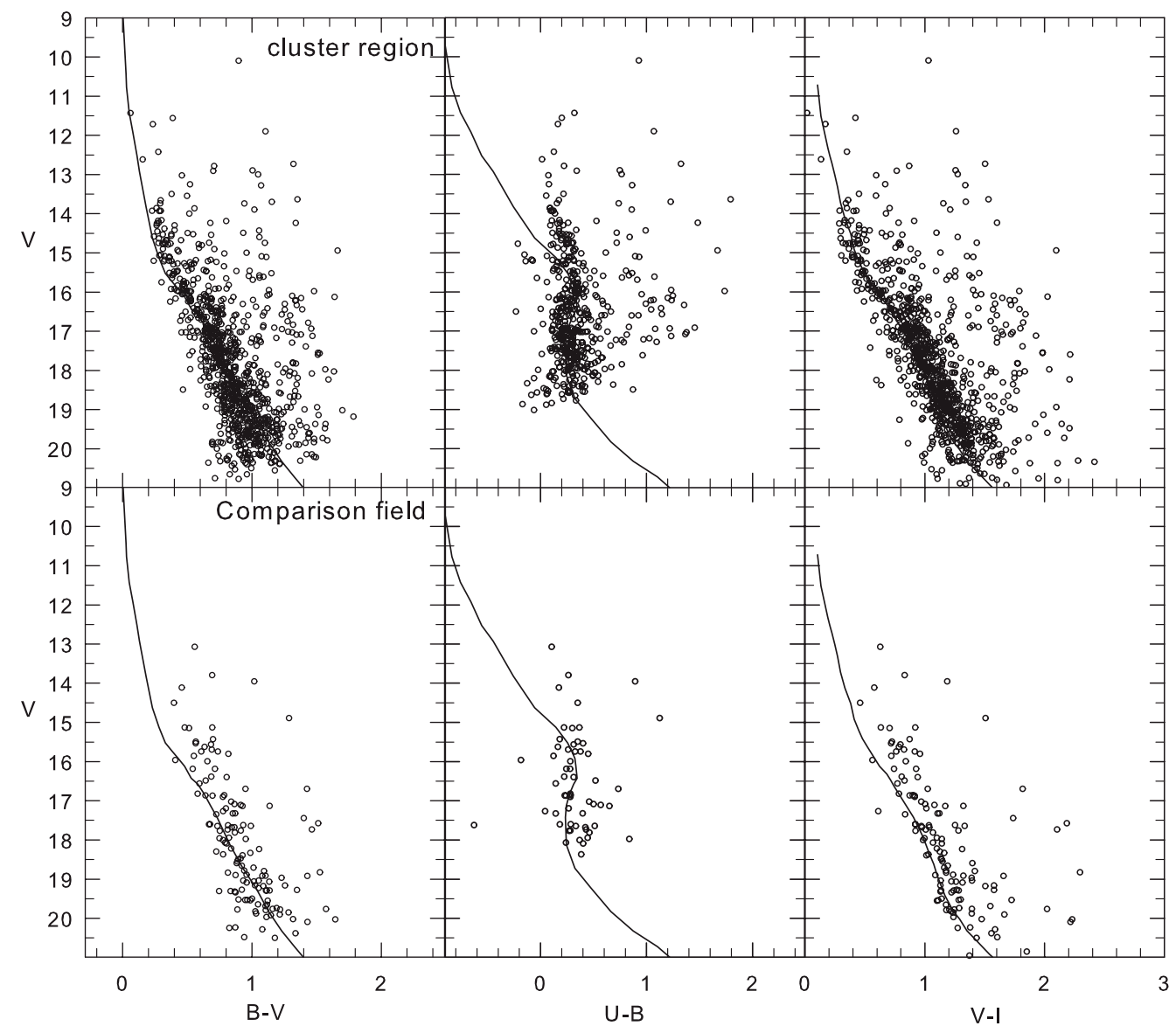

Fig. 5. Upper panel: The $V / B-V, V / U-B$, and $V / V-I$ diagrams of all stars inside the cluster radius. The Schmidt-Kaler (1982) ZAMS and the intrinsic locus of the $V / V-I$ sequence from Cousins (1978) adapted from the finding of $\S 3.2$ are shown as continuous lines. Lower panel: The same for the comparison field diagram

$V /(U-B)$ and $V /(V-I)$ color-magnitude diagrams, (CMD), respectively, of all stars inside the cluster limits $\left(<4.5^{\prime}\right)$; stars outside the cluster limit (field stars) were plotted too in a similar but not scaled diagrams. All figures show that Ruprecht 58 is a true cluster since the TCD shows a star sequence from $0.2<B-V<0.6$ sharing a common reddening value as indicated by the intrinsic line (Schmidt-Kaler, 1982) displaced $E_{B-V}=0.33 \pm 0.02$ and $E_{U-B}=0.29 \pm 0.04$. These stars, mainly late B- and A-type stars, constitute the upper main sequence of the cluster which extends from $V \simeq 13.5$ to $\simeq 17$. The $U-B / B-V$ field star diagram does not show such star sequence but a few stars of late A-types with similar reddening values what would indicate that for $r>4.5^{\prime}$ it is still possible to find some cluster members. Something similar happens in the CMDs for the cluster and the comparison areas where the potential cluster members clearly emerge for $V<17$. The visual inspection reveals too a number of stars in the CMDs that probably belong to an evolved 
field star population.

To assess memberships in Ruprecht 58 we consider that the TCD shows reddening values for the bright probable members going from $E_{B-V}=0.25$ to $E_{B-V}=0.41$ approximately. Therefore, all the stars falling outside that reddening limits are assumed cluster non members. The stars inside the reddening limits were then fitted using the Schmidt-Kaler (1982) ZAMS and mean reddening values $E_{B-V}=0.33$ and $E_{U-B}=0.29$. A careful comparison of the position of every star in each diagram was made again to reject out those ones located $1.3 \mathrm{mag}$ above the ZAMS and $0.3 \mathrm{mag}$ below it. This way we excluded evolved late-type and/or main sequence field stars of foreground without eliminating probable binaries. This method works reasonably well for main sequence stars brighter than $V \approx 17$ but it becomes uncertain longwards that magnitude where the contamination by field interlopers may be of some relevance. Despite this, they will cause no alteration of the cluster parameters when computed with stars down to $V \approx 17$. Some bright yellow/red stars at the top of the CMDs are not easy to reject out as they could be late evolved cluster members. To clarify this point we need first to derive the cluster distance and then to apply the spectroscopic parallax method using the spectral classification we have already done.

Table 3

Spectral types of stars in the area of Ruprecht 58

\begin{tabular}{rlccrrrrl}
\hline No. & S.T. & \multicolumn{1}{c}{$V$} & $(B-V)$ & $(U-B)$ & $E_{(B-V)}$ & $M_{V}$ & $d_{\odot}[\mathrm{pc}]$ & Comments to S.T. \\
\hline 1 & G8III & 10.09 & 0.89 & 0.92 & $(0.00)$ & 0.8 & 721 & \\
2 & B9IV & 11.43 & 0.06 & 0.31 & 0.13 & -0.2 & 1762 & \\
4 & F0III" & 11.56 & 0.38 & 0.20 & 0.18 & 1.5 & 794 & k A7V-H F0III \\
5 & G8III & 11.89 & 1.10 & 1.07 & 0.16 & 0.8 & 1312 & \\
6 & A0III & 12.42 & 0.27 & 0.12 & 0.31 & 0.0 & 1986 & \\
9 & G2III & 12.78 & 0.70 & 0.22 & $(0.00)$ & 0.9 & 2377 & \\
13 & F8Vp* & 13.02 & 0.46 & 0.07 & $(0.00)$ & 4.0 & 637 & k F0V-Gband F5V-H F8V \\
15 & G0Vp* & 13.25 & 0.52 & 0.08 & $(0.00)$ & 4.4 & 589 & k F2V-Gband F5V-H G0V \\
16 & G5III & 13.28 & 1.07 & 0.86 & 0.21 & 0.9 & 2218 & \\
17 & F0Vp* & 13.50 & 0.38 & 0.22 & 0.08 & 2.7 & 1288 & k A3V-H F0V \\
18 & F8Vp* & 13.55 & 0.50 & 0.05 & $(0.00)$ & 4.0 & 813 & k F0V-Gband F5V-H F8V \\
20 & A1IV & 13.66 & 0.29 & 0.17 & 0.28 & 0.7 & 2618 & \\
21 & G8III & 13.70 & 1.15 & 1.23 & 0.21 & 0.8 & 2818 & \\
\hline
\end{tabular}

Note: $E_{(B-V)}$ and $M_{V}$ come from the color index and absolute magnitude according to the star spectral type (Schmidt-Kaler 1982); for peculiar stars $M_{V}$ and $E_{(B-V)}$ correspond to the spectral type classification using hydrogen lines

To compute the cluster distance, fitting the Schmidt-Kaler (1982) ZAMS, we need to analyze first the extinction law, $R=A_{V} / E_{(B-V)}$, towards the cluster using the $(B-V)$ vs $(V-I)$ plots of all the probable cluster members as shown in Fig. 6. It is simple to see that cluster members tightly follow the galactic reddening vector $E_{(V-I)} / E_{(B-V)}=1.24$ (Dean et al. 1978) suggesting then that the extinction law is a normal one with $R=3$.1. If $R$ is normal the mean visual absorption in the zone turns out to be $\left[A_{V}=3.1 \times\left\langle E_{(B-V)}\right\rangle=1.02 \pm\right.$ 0.18]; the reddening-free colors and $V_{0}$ magnitudes of cluster members can be immediately obtained by correcting the observed colors with the average color 


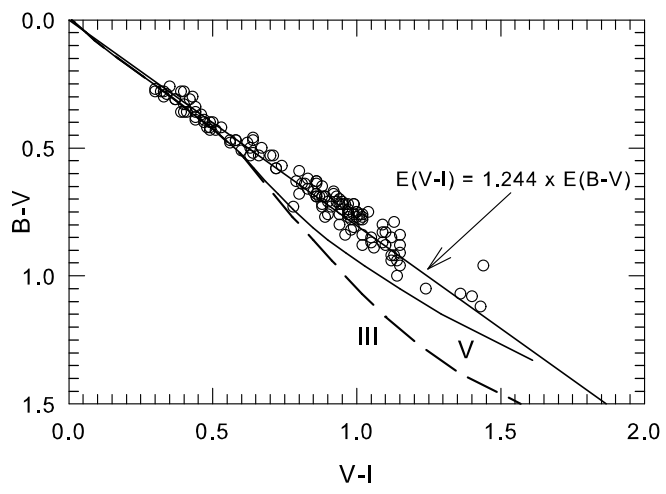

Fig. 6. The cleaned TCD, $B-V$ vs $V-I$, of stars in the zone of Ruprecht 58 . Intrinsic lines for luminosity classes, $\mathrm{V}$-continuous- and III -dashed-, and the relation given by Dean et al. (1978) are also indicated

excesses given above, and the computed visual absorption, $A_{V}$. The ZAMS fitting shown in Fig. 5 was carried out on the three CMDs [for the $V_{0} / V-I$ intrinsic locus it has been adapted from the correspondence of colors and spectral types given by Cousins (1978) and Schmidt-Kaler (1982)]. The mean of the fittings yielded a reddening-free distance modulus of $V_{0}-M_{V}=12.96$ which locates the cluster at $3.9 \mathrm{kpc}$ from the Sun. If we assume a 0.2 error of the fitting (from eye-inspection) and it is added quadratically to the uncertainties in color excess and visual absorption, the distance to the cluster may be wrong by no more than $\pm 400 \mathrm{pc}$.

We can now apply the spectroscopic parallax method using the intrinsic colorspectral type correspondence as given in Schmidt-Kaler (1982) to secure the membership of the bright red/yellow stars mentioned above. Some of these stars show a few hundredths of negative $E_{B-V}$ (indicated with brackets in Table 3) which are explained by spectral sub-type uncertainties and the few amount of reddening undergone by them. Values given in column 8, Table 3 , show that these stars are not cluster members. This is, the spectroscopic classification shows that bright yellow/red stars are foreground to the cluster. A question arises about stars No. 20 (A1IV) and 21 (G8III) that are the farthest stars $(d>2.6 \mathrm{kpc})$ and could still be members of the cluster in case of a probable variation of the stellar sub-type. We find this hard to happen as for the giant G8III and the A1IV stars, the absolute magnitudes would vary no more than $0.2-0.3$ respectively. These variations are not important enough to get these stars close to the cluster. Column 12, Table 2, remarks the photometric membership that are adopted in this article.

As for the cluster age, we measured in Fig. 7 the locations of the red and blue "turn-off", TOs -see Figs. 5b, 5d, and 5e of Meynet et al. (1993)- which were set at $M_{V} \approx 0.0 \ldots 0.4$ and $(B-V)_{0} \approx 0.0 \ldots 0.03$ respectively. They give an age of $250 \pm 120 \mathrm{Myr}$. We also superposed isochrones from a set of evolutionary 


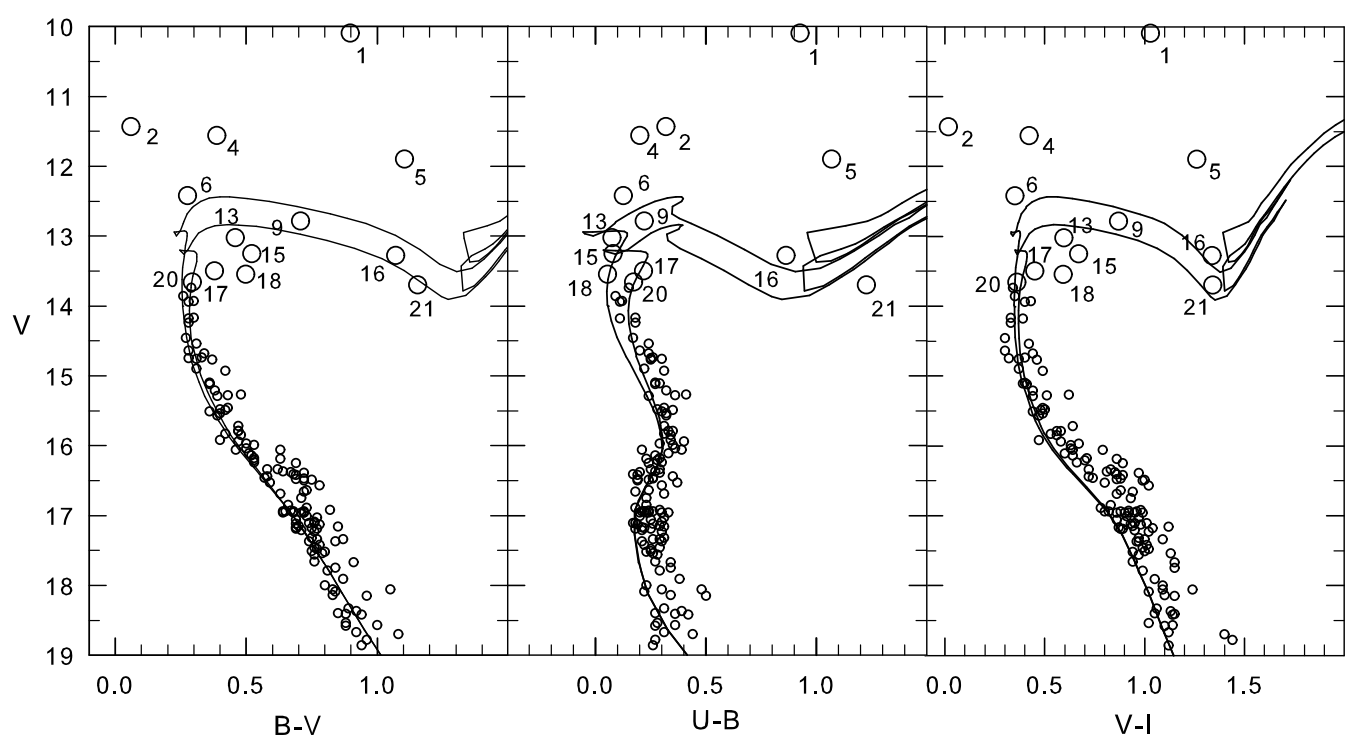

Fig. 7. The cleaned CMDs of the cluster. Small open circles are the probable cluster members from photometry and spectroscopy. Big open circles and numbers indicate stars with spectral types. Continuous lines are the isochronous curves for 250 and $300 \mathrm{Myr}$, from above to below respectively

tracks computed with mass loss and overshooting by Girardi et al. (2000); some spread at the upper main sequence stars makes it difficult to assign a unique age to this cluster. Therefore the cluster age ranges from 250 to 300 Myr. It is to be noticed from Fig. 7 that the number of binaries among cluster members could be high producing thus an artificial age spread and making this cluster younger than it is.

\section{Confirming the nature of Ruprecht 58}

Another way to confirm the cluster reliability comes from UCAC2 (Zacharias et al. 2004) proper motion catalog containing 1859 stars in a $20^{\prime}$ radius from the center of Ruprecht 58. The catalog precision in the star positions goes from about 20 mas for stars in the range $10<V<14$ to about 70 mas at $V \approx 16$ while proper motion errors go from 1-3 mas/yr down to $V \approx 12$ and 4-7 mas/yr at fainter magnitudes. Fig. 8 (left panel) shows the proper motionvector point diagram of 518 stars in a $10^{\prime}$ radius where 126 of them -shown with filled squares- have photometry obtained by us. The right panel of the figure shows the $V$ distribution of stars with photometry and proper motions contrasted with stars having only photometry. Since longwards $V>15.5$, the catalog shows evident signs of incompleteness, our analysis is seriously restricted to the bright stars of the sample. A detailed description of the method 

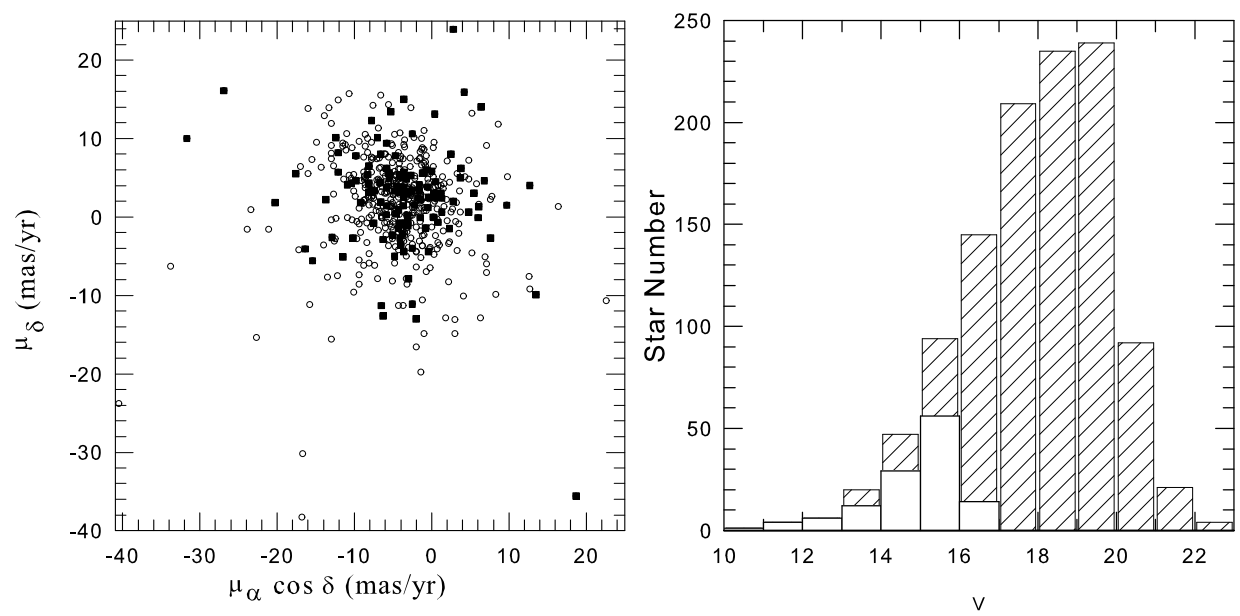

Fig. 8. Left: The proper motion-vector point diagram of 518 stars in the cluster area. Filled squares are stars with proper motions and photometry. Small open circles for the remaining stars. Right: The magnitude distribution of all stars in our sample (hatched histogram) and of stars with proper motions and photometry (white histogram)

we are using can be found in de Elia (2004). In short, we determine cluster memberships using a variation of the Vasilevskis et al. (1958) method who proposed a mathematical model where an elliptical bivariate normal frequency distributions and another circular one, for field and cluster stars respectively, describe the problem entirely. Since the parameters of the distributions are function of star magnitudes and the angular size of the selected field, the membership probabilities may be overestimated for stars far from the cluster center and underestimated for stars near it while underestimated for bright stars and overestimated for the faint ones. Jones \& Walker (1988) improved the method using an exponential function to describe the areal star density for the cluster stars according to Van den Bergh \& Sher (1960). As shown below, the method requires that nine unknown parameters be simultaneously fitted using the method of maximum likelihood (Sanders 1971). Both distributions are described by:

$$
\Phi_{c_{i}}\left(\mu_{x_{i}}, \mu_{y_{i}}, V_{i}, r_{i}\right)=\frac{\rho_{c}\left(V_{i}, r_{i}\right)}{2 \pi \sigma^{2}} \exp \left[-\frac{\left(\mu_{x_{i}}-\mu_{x_{c}}\right)^{2}+\left(\mu_{y_{i}}-\mu_{y_{c}}\right)^{2}}{2 \sigma^{2}}\right]
$$

for cluster stars and

$$
\Phi_{f_{i}}\left(\mu_{x_{i}}, \mu_{y_{i}}, V_{i}\right)=\frac{\rho_{f}\left(V_{i}\right)}{2 \pi \sigma_{x} \sigma_{y}} \exp \left[-\frac{\left(\mu_{x_{i}}-\mu_{x_{0}}\right)^{2}}{2 \sigma_{x}^{2}}-\frac{\left(\mu_{y_{i}}-\mu_{y_{0}}\right)^{2}}{2 \sigma_{y}^{2}}\right],
$$


for field stars; where $\mu_{x_{0}}, \mu_{y_{0}}$ denote the centroid of the field stars; $\mu_{x_{c}}, \mu_{y_{c}}$ the centroid of the cluster stars; $\sigma_{x}, \sigma_{y}$ eliptical dispersions; $\sigma$ circular dispersion; $\mu_{x_{i}}, \mu_{y_{i}}, r_{i}, V_{i}$ are the proper motions, the distance from the cluster center and the magnitude of the i-th star. Besides,

$$
\rho_{c}\left(V_{i}, r_{i}\right)=\rho_{0}\left(V_{i}\right) e^{-\frac{r_{i}}{r_{0}\left(V_{i}\right)}}
$$

with $\rho_{c}\left(V_{i}, r_{i}\right)$ to describe the areal cluster star density as a function of $V_{i}$ and $r_{i} ; \rho_{0}\left(V_{i}\right)$ is the central surface density, $r_{0}\left(V_{i}\right)$ the characteristic radius; the areal density for the field stars is described by $\rho_{f}\left(V_{i}\right)=f_{0}\left(V_{i}\right)$ where $f_{0}\left(V_{i}\right)$ only depends on magnitudes. $N_{c}$, the number of cluster members, is obtained from $N_{c}=2 \pi \rho_{0}\left(V_{i}\right) r_{0}\left(V_{i}\right)^{2}$. The dynamical membership probabilities for i-th star can be calculated as follow:

$$
P_{i}=\frac{\Phi_{c_{i}}}{\Phi_{c_{i}}+\Phi_{f_{i}}}
$$

The star sample was subdivided into four groups of $V, V_{1}<13.5,13.5<$ $V_{2}<14.5,14.5<V_{3}<16.0$ and $V_{4}>16.0$. The criterion to group stars this way is that each sub-sample must be enough populated for a better statistical analysis. Finally, we stated that stars with $P_{i}>0.40$ are probable dynamical members. The result of the method applied to groups $V_{2}$ and $V_{3}$ is given in Table 4; the last column of Table 2 gives the computed probabilities for each of the 126 stars.

Table 4

Cluster and field star parameters

\begin{tabular}{c|cccccc}
\hline \multicolumn{7}{c}{ Cluster } \\
\hline$V_{i}$ & $N_{c}$ & $r_{0}[$ arcmin $]$ & $\rho_{0}\left[\right.$ arcmin $\left.^{-2}\right]$ & $\mu_{x_{c}}[$ mas $/ y]$ & $\mu_{y_{c}}[$ mas $/ y]$ & $\sigma[$ mas $/ y]$ \\
\hline$V_{2}$ & 13 & 1.67 & 0.73 & -2.77 & 4.54 & 1.64 \\
$V_{3}$ & 47 & 3.54 & 0.60 & -2.70 & 3.19 & 2.16 \\
\hline \multicolumn{7}{c}{ Field } \\
\hline$V_{i}$ & $f_{0}\left[\right.$ arcmin $\left.^{-2}\right]$ & $\mu_{x_{0}}[$ mas $/ y]$ & $\sigma_{x}[\text { mas } / y]_{y}$ & $\mu_{y_{0}}[$ mas $/ y]$ & $\sigma_{y}[$ mas $/ y]$ \\
\hline$V_{2}$ & 0.25 & -4.34 & 5.44 & 3.02 & 4.21 \\
$V_{3}$ & 0.65 & -4.17 & 5.18 & 1.91 & 4.19 \\
\hline
\end{tabular}

It is worth mentioning that the characteristic radius $r_{0}\left(V_{i}\right)$, increases with the magnitude; therefore, bright cluster members are much more concentrated than the faint ones. The proper motion dispersion also increases for faint magnitudes, but not so clearly as $r_{0}$ does.

For group $V_{1}$, as seen in Table 2, the cluster has no dynamical members brighter than $V=13.5$. This result is entirely consistent with the spectrophotometric result that shows no member above $V=13.5$. The situation is, on the other hand, completely uncertain for $V_{4}$ star group $[V>16.0]$. A reading of Table 2 gives: twenty one stars are simultaneously dynamical and photometric members; eighteen stars are photometric members but not astrometric 
members; fifty eight stars are neither photometric nor astrometric members and twenty five stars that are dynamical members were found non-members from the photometric analysis.

Table 4 shows that at $1 \sigma$ the field and cluster distributions do not differ substantially. However, it is worth mentioning that the study of proper motions and photometry/spectroscopy show a definite coincidence for the brightest stars which have been found not to be cluster members. Certainly, errors in the proper motion measures and the huge un-completeness of the sample make the dynamical membership estimate rather unstable below $V=13.5 \ldots 14$. Notwithstanding, star counts and the proper motion study give both a similar -increasing towards faint magnitudes- cluster size. That is why, in the next section, the cluster mass spectrum will be determined only with photometric members.

\section{The cluster mass spectrum}

We shall build now the cluster mass spectrum, defined as the number of stars found per mass interval. In particular, for all the stars below the cluster TO, the mass spectrum reflects the initial mass function, IMF, if we assume all the star formed at a same time. In the present case bright (most massive) members are easy to identify; but it is not a simple task to find all the faint (less massive) members as they are mixed with field interlopers. However, field interloper effect has been minimized in the best possible way so that we assume the apparent luminosity function, LF, of the cluster [constructed with only photometric members] is trustable down to $V \approx 17$ and can be converted into the cluster mass spectrum.

Table 5

Luminosity function and mass spectrum of Ruprecht 58

\begin{tabular}{rrrc|cccc}
\hline$\Delta M_{V}$ & $\mathrm{~N}$ & $\log m$ & $\log \left(\frac{d N}{\Delta \log m}\right)$ & $\Delta M_{V}$ & $\mathrm{~N}$ & $\log m$ & $\log \left(\frac{d N}{\Delta \log m}\right)$ \\
\hline \hline$-0.5 \ldots 0.0$ & 6 & 0.59 & 1.90 & $2.5 \ldots 3.0$ & 21 & 0.19 & 2.57 \\
$0.0 \ldots 0.5$ & 7 & 0.51 & 1.99 & $3.0 \ldots 3.5$ & 23 & 0.14 & 2.63 \\
$0.5 \ldots 1.0$ & 13 & 0.44 & 2.27 & $3.5 \ldots 4.0$ & 10 & 0.09 & 2.28 \\
$1.0 \ldots 1.5$ & 10 & 0.38 & 2.18 & $4.0 \ldots 4.5$ & 11 & 0.03 & 2.36 \\
$1.5 \ldots 2.0$ & 19 & 0.31 & 2.46 & $4.5 \ldots 5.0$ & 6 & -0.01 & 2.12 \\
$2.0 \quad . .2 .5$ & 25 & 0.25 & 2.62 & $5.0 \ldots 5.5$ & 2 & -0.05 & 1.68 \\
\hline
\end{tabular}

The apparent LF was converted into the true LF using the cluster distance modulus and the visual absorption given above to obtain individual $M_{V} \mathrm{~s}$. The final step was to apply the mass-luminosity relation from Scalo (1986) to each luminosity bin in the true LF (see Table 5) to derive the stellar mass distribution shown by the mass points in Fig. 9. This is a probed and straightforward method to transform the LF into the mass spectrum for all stars below the cluster TO when the range of star formation time is less than the cluster age 
(Phelp \& Janes 1993, 1994). Since the mass spectrum is defined by the number of stars counted in the mass range $m \pm \Delta m / 2$, the slope $x$ of the mass distribution can be computed by:

$$
x=-\frac{\log (d N / \Delta \log m)}{\log m}
$$

assuming the mass spectrum is represented by a power law.

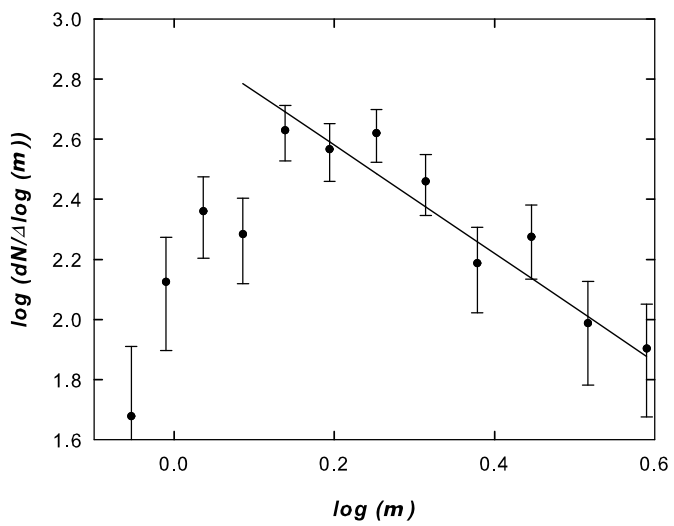

Fig. 9. The mass function of Ruprecht 58. Error bars depict $\sqrt{N}$ of the counts at a given mass bin. Solid line is the slope $x=1.8$ of the fitting

To derive the slope of the mass spectrum we applied a statistical-weighted least squares fit to all the mass points in Fig. 9 (covering the magnitude range $13.5<V<17.25$ ) except for the last four bins that surely are affected by incompleteness. Notice that this apparent magnitude range is the one of maximum certainty on memberships from a photometric and spectroscopic point of view. The fitting yielded a slope $x=1.8 \pm 0.2$, a value higher than the standard one determined by Salpeter (1955) but of the same order of the typical values found by Tarrab (1982) for clusters of about 300 Myr. Using "only" stars located in the cluster main sequence the mass spectrum slope is free from evolutionary effects. No matter the presence of interlopers, the construction of the mass spectrum of any cluster may undergo other type of strong uncertainties such as the presence of accretion disks (probable of no importance in clusters this age), differential reddening and unresolved multiple systems that spoil the results; but no doubt, the net effect due to unresolved binaries (Scalo 1986) is the most difficult to assess: if the mass spectrum of the secondary stellar components is similar to that of primary stars then their influence on the computed slope is negligible (Vanbeveren 1982); however, detailed analysis made by Sagar \& Richtler (1991) demonstrate that undetected binaries can rise the slope of a mass function by 0.25 for a binary percentage of $50 \%$. In the present case, allowing for fitting errors, the slope we found is 
close to the upper limit of the $x$ range, $-1.7 \pm 0.5$ to $-1.3 \pm 0.5$, valid for the mass range from $1-10 m_{\odot}$ as described by Scalo (1998).

\section{Concluding remarks}

For the first time a CCD $U B V I$ photometric survey plus spectral classification have been done in the area of Ruprecht 58 to reveal its fundamental parameters. Our results find additional support in a proper motion analysis performed with data taken from UCAC2.

The cluster angular size from star count is close to $9^{\prime}$ corresponding to a linear size of $10 \mathrm{pc}$ approximately. The mean cluster reddening values are $E_{(B-V)}=$ 0.33 and $E_{(U-B)}=0.29$. The absorption law towards it is normal with a mean visual absorption of $A_{V}=1.02$. The cluster is then located at a distance of $3.9 \mathrm{kpc}$ and its age ranges from 250 to $300 \mathrm{Myr}$ according to Girardi et al. (2000) isochrone curves. It is placed in a galactic zone containing about 60 more clusters with distances between 1 and $5 \mathrm{kpc}$, with a peak from 1 to $3 \mathrm{kpc}$. There are also several HiI regions detected in the optical and radio, and 7 Wolf-Rayet stars mostly of C-type (van der Hucht 2001) - all in the first 4 kpc from the Sun. In particular, $2^{\circ}$ eastwards Ruprecht 58, two Hit regions, RCW 19 and 20 (H109 $\alpha$ source 253.6-0.20) appear at kinematic distances of $3.1 \mathrm{kpc}$ (Georgelin \& Georgelin 1976), i.e. in front of the cluster and therefore with no spatial relation to it. All this would indicate that the cluster is placed along the Local-arm, probably in the conjunction with the internal edge of the Perseus spiral-arm (Carraro et al. 2005b; Moitinho et al. 2006).

The slope of the cluster mass spectrum is $x=1.8$, steeper than the typical mass spectrum of field stars (Salpeter 1955) but comparable and even flatter than the average of mass spectrum slopes found by Tarrab (1982) for cluster with ages ranging from 300 to $600 \mathrm{Myr}$ and reasonably close to the slope values proposed by Scalo (1998) for the mass range 1 to $10 m_{\odot}$.

This publication makes use of data from the Two Micron All Sky Survey funded by the National Aeronautics and Space Administration and the National Science Foundation. This article is partially based in the Digitized Sky Survey.

$E G, R A V, G R S$ and RBO acknowledge the financial support from the PIP 5970 (CONICET) and to the IALP-CONICET. Special thanks are given to the CASLEO staff for the technical support. 


\section{References}

[1] Baume G., Moitinho A., Giorgi E., Carraro G., Vázquez R.A. 2004 A\&A, 417, 961

[2] Becker W. \& Fenkart R. 1970, IAU Symposium 38, The Spiral Structure of the Galaxy, eds. W. Becker \& G. Contopoulos (Dordrecht: Reidel), p. 205

[3] Bellazzini M., Ibata R.A., Monaco L., Martin N., Irwin M.J., Lewis G.F., 2004, MNRAS 354, 1278

[4] Carraro G., Baume G., Piotto G., Méndez R.A., Schmidtobreok K. 2005a, A\&A, 436, 527

[5] Carraro G., Vázquez R.A., Moitinho A. \& Baume G.L., 2005b, ApJ, 630, L153L156,

[6] Cousins, A.W.J. 1978, MNASSA, 37, 62

[7] Dean J.F., Warren P.R., \& Cousins A.W.J. 1978, MNRAS, 183, 569

[8] de Elía, G.C. 2004 Tesis de Licenciatura, Fac. de Ciencias Astronómicas y Geofísicas, Univ. Nac. de La Plata

[9] Feinstein A., 1994, Rev. Mex. Astron. Astroph. 29, 141

[10] Georgelin, Y.M., \& Georgelin, Y.P. 1976, A\&A, 49, 57

[11] Giorgi, E.E., Vázquez R.A., Baume G., Seggewiss W., \& Will J.-M. 2002, A\&A, 381,884

[12] Giorgi, E.E., Baume G., Solivella G., Vázquez R.A. 2005, A\&A, 432, 491

[13] Girardi, L., Bressan, A., Bertelli, G., \& Chiosi, C. 2000, A\&AS, 141, 371

[14] Janes, K., \& Adler, D. 1982, ApJS, 49, 425

[15] Jones, B.F. \& Walker, M.F. 1988, AJ 95, 1755

[16] Landolt, A.U. 1992, AJ, 104, 340

[17] Lindoff, U. 1968, Arkiv fur Astron., 4, 587

[18] Martin N., Ibata R.A., Bellazzini M., Irwin M.J., Lewis G.F., Dehnen W., 2004, MNRAS 348, 12

[19] Massey, P., Johnson K.E., \& DeGioia-Eastwood K. 1995, ApJ, 454, 151

[20] May J., Alvarez H., Bronfman L., 1997, A\&A 327, 325

[21] Meynet, G., Mermilliod, J.-C., \& Maeder A. 1993, A\&AS, 98, 477

[22] Moitinho, A. 2001, A\&A, 370, 436 
[23] A. Moitinho, R. A. Vázquez, G. Carraro, G. Baume, E. E. Giorgi and W. Lyra, 2006 MNRAS, 368, L77

[24] Momany Y., Zaggia S.R., Bonifacio P., Piotto G., De Angeli F., Bedin L., Carraro G., 2004, A\&A 421, L29

[25] Phelps, R.L., \& Janes, K.E. 1993, AJ, 106, 1870

[26] Phelps, R.L., \& Janes, K.E. 1994, ApJS, 90, 31

[27] Russeil D., 2003, A\&A 397, 133

[28] Salpeter, E.E. 1955, ApJ, 121, 161

[29] Sagar, R., \& Richtler, T. 1991, A\&A, 250, 324

[30] Sanders, W.L. 1971, A\&A 14, 226

[31] Scalo, J. 1986, Fundam. Cosmis Phys., 11, 1

[32] Scalo, J. 1998, in The Stellar Initial Mass Function (38th Herstmonceux Conference), ed. G. Gilmore, \& D. Howell, ASP Conf. Ser., Vol. 142, p. 201

[33] Schmidt-Kaler, Th. 1982, in Landolt-Börnstein, N.S., VI/2b, Springer, BerlinHeidelberg-New York, p. 1

[34] Stetson, P.B. 1987, PASP, 99,191

[35] Tarrab, I. 1982, A\&A, 109, 285

[36] Vanbeveren, D. 1982, A\&A, 115, 65

[37] van den Bergh,S. and Sher, D. (1960), Publ. David Dunlap Obs, 2, 203.

[38] van der Hucht K.A. 2001, New Astron. Rev. 45, 135

[39] Vasilevskis, S., Klemola, A., Preston, G. 1958, AJ 63, 387

[40] Zacharias N., Urban S.E., Zacharias M.I., Wycoff G.L., Hall D.M., Monet D.G., Rafferty T.J. 2004, AJ 127, 3043 